\title{
Atomic Force Microscopy for the Evaluation of Corneal Surface Roughness after Femtosecond Laser Flap Creation and Excimer Ablation.
}

Rafael Cañones-Zafra ( $\nabla$ rafael.canones@gmail.com )

Hospital Universitario Príncipe de Asturias: Hospital Universitario Principe de Asturias https://orcid.org/0000-0002-5353-0297

Juan Gros-Otero

Clinica Rementeria

Montserrat Garcia-Gonzalez

Clinica Rementeria

\section{Santiago Casado}

Universidad Técnica de Ambato: Universidad Tecnica de Ambato

\section{Samira Ketabi}

Hospital Universitario Príncipe de Asturias: Hospital Universitario Principe de Asturias

Cesar Villa-Collar

Universidad Europea de Madrid Campus de Villaviciosa de Odón: Universidad Europea de Madrid SLU

Miguel A. Teus

Hospital Universitario Príncipe de Asturias: Hospital Universitario Principe de Asturias

\section{Research Article}

Keywords: Femtosecond Laser, Corneal Roughness, Atomic Force Microscopy

Posted Date: December 21st, 2021

DOI: https://doi.org/10.21203/rs.3.rs-1083083/v1

License: (c) (i) This work is licensed under a Creative Commons Attribution 4.0 International License.

Read Full License 


\section{Abstract}

Introduction: It is well known that the femtosecond laser lamellar cut has some degree of roughness. Nevertheless, as in femtosecond laser assisted LASIK (FS-LASIK), an excimer LASIK ablation is performed, the post-ablation stromal bed should show a marked decrease in roughness. We decided to compare, using atomic force microscopy (AFM), the roughness of the corneal stromal bed, after a Femtosecond lasers device (FS) flap was created with or without an excimer myopic ablation.

Methods: Using 6 freshly enucleated porcine eyes, we created in every eye a flap using a femtosecond laser. Additionally in 3 eyes an excimer laser ablation to correct -3 diopters (D) was made. AFM imaging of the remaining corneal stroma was performed. Ten different square areas of $20 \mu \mathrm{m} \times 20 \mu \mathrm{m}$ at the central area of the stroma of each corneal sample were studied. The roughness parameters used was the root-mean-square (RMS) deviation from a perfectly flap plane.

Results: The RMS deviation were $360 \pm 120 \mathrm{~nm}$ in femtosecond laser only, and $110 \pm 20 \mathrm{~nm}$ in those cases where excimer is also involved $(p<0.0001)$.

Conclusions: Our results show that the roughness of the surface treated with excimer is clearly lower than in the group with no excimer ablation, thus the application of laser excimer after a flap creation by femtosecond laser flap creation may soften the nano-irregularities created by this technique.

\section{Introduction}

The roughness of the stromal surfaces obtained following femtosecond (FS) laser-assisted keratomileusis (LASIK) has been recognized as a clinically relevant issue, because it is associated with optical aberrations and dysphotopic phenomena ("rainbow glare") that may compromise patients' quality of vision in the postoperative period ${ }^{1}$.

Nowadays, it is possible to assess the characteristics of corneal tissue planes by means of three different strategies: at a near-macroscopic scale using anterior segment optical coherence tomography $(\mathrm{OCT})^{2}$, at a microscopic scale using scanning electron microscopy (SEM) and transmission electron microscopy $(T E M)^{3}$, and in a nano-scale (i.e. with a precision in the range of few nanometers) using atomic force microscopy (AFM) $)^{1,4-5}$.

Femtosecond laser devices play an increasingly important role in modern refractive surgery and are currently preferred to mechanical microkeratomes for flap creation ${ }^{6}$. Furthermore, FS laser-assisted refractive lenticule extraction (ReLEx) has been proposed as an alternative to laser excimer ablation for the correction of myopia ${ }^{7-8}$. However, it is well known that the plane of the FS lamellar cut is not absolutely smooth ${ }^{5-9}$. In fact, the corneal tissue plane has a certain degree of roughness, mostly because the tissue-cutting laser spots are separated from one another (the so called "spot distance") ${ }^{5-9}$. Recent efforts have focused on the optimal FS laser parameters that would ensure a smoother interface ${ }^{9}$. 
Nevertheless, because in FS-LASIK procedures an excimer ablation is performed over the corneal stroma, the post-ablation stromal bed might be expected to show a decrease in roughness.

The aim of the current study was to evaluate the smoothening effect of excimer ablation on corneal tissue planes of freshly enucleated porcine eyes that underwent LASIK flap creation using a FS laser. Using AFM, the stromal bed roughness of eyes that underwent FS LASIK flap creation alone was compared to that of eyes that in addition underwent excimer ablation following the FS LASIK flap creation.

\section{Materials And Methods}

\section{Procedure}

We designed an experimental comparative study using six freshly enucleated porcine eyes. Within 24 hours from the enucleation, the same experienced surgeon (JGO) created a lamellar corneal flap using the $60 \mathrm{KHz}$ IntraLase ${ }^{\circledR}$ FS laser (Abbott Medical Optics, Inc., Santa Ana, CA, USA). The following parameters were used: flap depth 110 microns, flap diameter $9.00 \mathrm{~mm}$, hinge position 90 degrees (i.e., superior), hinge angle: 50 degrees, side-cut energy $0.95 \mathrm{~mJ}$, side-cut angle 135 degrees. Pocket depth and width were set at $230 \mu \mathrm{m}$ and $0.23 \mu \mathrm{m}$, respectively, with $5 \mu \mathrm{m}$ tangential and radial spot separations. Immediately after the procedure, the flap was lifted and then repositioned using a LASIK spatula. Next, myopic excimer laser ablation to correct -3.0 diopters (D) was performed in three of the six eyes using a WaveLight Allegretto EyeQ 400-Hz laser (Alcon Laboratories, Inc. Fort Worth, TX, USA). The laser parameters of the ablation were: optical zone: $6,5 \mathrm{~mm}$, transition zone: $0,3 \mathrm{~mm}$, ablation zone: $7,10 \mathrm{~mm}$, maximum central ablation: 45,5 microns. When the above procedures were completed, a $9 \mathrm{~mm}$ trephination of the cornea was performed with a Barron vacuum donor cornea punch (Katena Inc, Denville, NJ, USA) in all eyes. Immediately after the trephination, the flap was lifted and amputated with scissors and the globes were prepared for AFM analysis of the stromal bed roughness. The endothelial side of the corneal button obtained was glued with cyanoacrylate adhesive to a microscope slide and covered with $2.5 \%$ glutaraldehyde solution for 24 hours before AFM measurements of the stromal bed roughness were performed.

Ten randomly selected areas from each sample were studied, thus a total of 30 regions from each group were analyzed.

\section{Imaging}

AFM imaging was performed using a JPK NanoWizard IIß atomic force microscope coupled to a Nikon Eclipse Ti-U inverted optical microscope, in contact mode immersed in liquid, and employing Olympus OMCL-RC800PSA commercial silicon nitride cantilever tips $(0.05 \mathrm{~N} / \mathrm{m}, 18 \mathrm{kHz})$, with typical $15 \mathrm{~nm}$ radius at the end. Vertical accuracy of the instrument is in the order of $0.1 \mathrm{~nm}$. 
Surface roughness measurements were made in 10 different square areas with dimensions $20 \mu \mathrm{m} x$ $20 \mu \mathrm{m}$ at the central part of each corneal sample, using a 512x512 point resolution. The images were processed and analyzed using the JPK Data Processing software. The roughness parameter used in this study was the root-mean-square (RMS) deviation from a perfectly flat plane within the analyzed area.

\section{Results}

The RMS (in microns) from AFM images of every sample analyzed was calculated. The results are depicted in Table 1.

Table 1

Results of the RMS (in microns) from AFM images of every sample analyzed.

\begin{tabular}{|lllllll|}
\hline & FS-LASIK & & \multicolumn{5}{c|}{ FS-LASIK \& Excimer } \\
\cline { 2 - 3 } & $\underline{\text { Sample 2 }}$ & $\underline{\text { Sample 3 }}$ & $\underline{\text { Sample 4 }}$ & $\underline{\text { Sample 5 }}$ & Sample 6 \\
\hline Region 1 & 481 & 312 & 396 & 108 & 160 & 113 \\
\hline Region 2 & 208 & 312 & 414 & 108 & 132 & 130 \\
\hline Region 3 & 292 & 346 & 419 & 90 & 117 & 102 \\
\hline Region 4 & 202 & 337 & 672 & 92 & 120 & 122 \\
\hline Region 5 & 230 & 338 & 415 & 86 & 119 & 92 \\
\hline Region 6 & 288 & 277 & 408 & 69 & 138 & 85 \\
\hline Region 7 & 250 & 312 & 504 & 89 & 69 & 89 \\
\hline Region 8 & 278 & 376 & 558 & 90 & 82 & 114 \\
\hline Region 9 & 237 & 275 & 594 & 106 & 127 & 125 \\
\hline Region 10 & 509 & 258 & 358 & 96 & 150 & 148 \\
\hline Mean of each Sample & $\mathbf{2 9 7 . 5}$ & $\mathbf{3 1 4 . 3}$ & $\mathbf{4 7 3 . 8}$ & 93.4 & $\mathbf{1 2 1 . 4}$ & $\mathbf{1 1 2}$ \\
\hline
\end{tabular}

For the whole groups, the mean \pm standard deviation of the RMS values were $360 \pm 120 \mathrm{~nm}$ for the FSLASIK group, and $110 \pm 20 \mathrm{~nm}$ for the FS-LASIK and excimer group (P value=0.0001), (Figure 1).

Using atomic force microscopy we obtained three dimensional and vertical deflection images of the stromal surface of the FS-LASIK flap creation without excimer ablation group (Figure 2, Figure 3), and of the FS-LASIK flap creation and excimer laser ablation group (Figure 4, Figure 5).

\section{Discussion}


Our results show that excimer laser ablation following flap creation with an FS-LASIK does smoothen the nano-scale irregularities of the corneal stroma surface induced by the FS phototodisruption. This observation is clinically relevant, because the optical effects induced by a rough corneal surface after FSLASIK surgery may adversely affect the quality of vision in the postoperative period ${ }^{5-9}$.

Following its validation for ophthalmic purposes, AFM has become a method of choice for studying the roughness of various surfaces ${ }^{10}$. In fact, AFM is considered a more attractive option than SEM because it allows the acquisition of three-dimensional images of the surface of samples and offers the possibility of quantitative and objective analysis. An additional advantage of AFM over SEM is that the preparation of specimens for the former is simpler than for the latter. For these reasons, AFM is currently considered the gold standard for the study of stromal surface roughness ${ }^{5-11}$.

There are few reports on the use of AFM for the study of the stromal roughness after excimer laser ablation ${ }^{12-13}$. However, none of them analyzed the stromal roughness before and after the ablation. Instead, these experimental studies only examined the effect of the excimer laser ablation using different parameters for depth and energy following the mechanical removal of the epithelium ${ }^{12-13}$. Lombardo $M$ et al. were the first to study corneal roughness using AFM after a standard ablation using two different excimer lasers ${ }^{14}$, as well as the effect of a smoothening technique with viscoelastic application over the corneal stroma as a masking agent in photorefractive keratectomy ${ }^{4}$. In a later report, the same authors published the results of AFM analysis of the corneal epithelium and the photoablated corneal stroma ${ }^{1}$, but did not compare the roughness of the corneal stromal bed before and after the ablation.

Few investigators have used AFM to examine the effect of FS lasers on corneal surface roughness. Serrao et al studied the effect of different parameters on corneal surface roughness using the IntraLase platform for $\mathrm{LASIK}^{9-11}$, as well as the surface roughness and morphology of the posterior aspect of stromal lenticules created with a femtosecond laser (ReLEx) ${ }^{15}$. Ziebarth et al studied the quality of corneal lamellar cuts with the Visumax platform for FS-LASIK ${ }^{5}$. However, none of the aforementioned investigators performed an excimer ablation after the FS laser procedure to study the excimer-induced changes on corneal surface roughness.

To the best of our knowledge, the current study is the first to employ AFM for the assessment of the surface-smoothening effect induced by excimer ablation on a corneal tissue plane created by a FS laser. The fact that surface roughness decreases after the excimer ablation is fortunate, because it indicates that in real-life surgeries at least one of the surfaces created with the FS laser is smoothened.

Conceivably, in such cases the improved characteristics of the anterior surface of the stromal bed could translate to better quality of vision in the postoperative period.

Based on our results, one could consider those refractive techniques in which only a FS laser is used (e.g. ReLEx procedures). In these techniques the smoothening effect of excimer ablation is not present. Hence, in such procedures in addition to the roughening effect of the mechanical dissection of the lenticule, irregularities are added on both surfaces created by the FS laser ${ }^{16}$. This fact could explain the differences 
between LASIK and ReLEx in corrected distance visual acuity at 3 months after surgery in the Multicenter Prospective Cohort Study on Refractive Surgery in 15011 eyes ${ }^{17}$. This study clearly showed a lower gain of Snellen lines in eyes treated with ReLEx compared to those treated with LASIK, with a statistically significant difference in the loss of 1 Snellen line and in the proportion of eyes achieving so-called "supervision." Nonetheless, further studies with sufficiently long follow-up are needed to address these concerns.

A possible limitation of the present study is that the animal eyes that we used were operated within approximately 24 hours after enucleation. Consequently, post-mortem tissue changes may have interfered with the overall quality of the FS cut. In addition, only small areas $\left(20 \times 20 \mu m^{2}\right)$ of the corneal surface were studied with AFM, thus, morphologic features larger than the single area scanned do not contribute to the analysis.

The results of our study are not necessarily applicable to other commercially available femtosecond laser platforms, or even to the same platform with different settings. Nonetheless, our results clearly indicate the beneficial effect that excimer laser photoablation has on the surface roughness characteristics of tissue planes created with a FS laser.

\section{Conclusion}

Our results show that the roughness of the surface treated with excimer is clearly lower than in the group with no excimer ablation, thus the application of laser excimer after a flap creation by femtosecond laser flap creation may soften the nano-irregularities created by this technique.

\section{Declarations}

\section{Competing of interest:}

None of the authors has any financial or proprietary interest in any aspect of this study.

\section{Funding info:}

No private or public support was received for this study.

\section{Author contribution:}

All named authors meet the International Committee of Medical Journal Editors (ICMJE) criteria for authorship for this article, take responsibility for the integrity of the work as a whole, and have given their approval for this version to be published. 


\section{Data Availability:}

The datasets during and/or analyzed during the current study are available from the corresponding author on reasonable request

\section{Animal research:}

All procedures performed involving animals were in accordance with the ethical standards of the institution at which the study were conducted and ethical approval was obtained from COMITÉ ÉTICO DE INVESTIGACIÓN CLÍNICA REGIONAL DE LA COMUNIDAD DE MADRID (code 216/03, version 2.0 Mayo 2016).

The tables and figures are original and have been produced by the authors for this particular publication

\section{References}

1. Lombardo M, De Santo MP, Lombardo G, Barberi R, Serrao S (2006) Atomic force microscopy analysis of normal and photoablated porcine corneas. J Biomech 39:2719-2724

2. Jing Zhang MD, Yuehua Zhou MD, ChangbinZhai MD, Lei Tian MD (2013) Comparison of 2 femtosecond lasers for laser in situ keratomileusis flap creation.J Cataract Refract Surg; 39:922-927

3. Kymionis GD, Kontadakis GA, Naoumidi I, Kankariya VP, Panagopoulou S, Manousaki A, Grentzelos MA, Pallikaris IG (2014) Comparative study of stromal bed of LASIK flaps created with femtosecond lasers (IntraLase FS150, WaveLight FS200) and mechanical microkeratome. Br J Ophthalmol 98:133-137

4. Lombardo M, De Santo MP, Lombardo G, Barberi R, Serrao S (2005) Roughness of Excimer Laser Ablated Corneas With and Without Smoothing Measured With Atomic Force Microscopy. J Refract Surg 21(5):469-475Sep-Oct

5. Ziebarth NM, Dias J, Hürmeriç V, Shousha MA, Yau CB, Moy VT, Culbertson WW (2013) Yoo SH Quality of corneal lamellar cuts quantified using atomic force microscopy.J Cataract Refract Surg; 39:110-117

6. Shihao Chen MD, Yifan ODMSc, Feng MD, Aleksandar Stojanovic MD, Mirko R (2012 Jan) Jankov II, MD, PhD; Qinmei Wang, MD. IntraLase Femtosecond Laser vs Mechanical Microkeratomes in LASIK for Myopia: A Systematic Review and Meta-analysis.J Refract Surg. ; 28(1):15-24

7. Zhang Y, Shen Q, Jia Y, Zhou D, Zhou J (2016) Clinical Outcomes of SMILE and FS-LASIK Used to Treat Myopia: A Meta-analysis. J Refract Surg 32(4):256-265

8. Moshirfar M, McCaughey MV, Reinstein DZ, Shah R, Santiago-Caban L, Fenzl CR (2015) Smallincision lenticule extraction.J Cataract Refract Surg. ; 41:652-665

9. Serrao S, Buratto L, Lombardo G, De Santo MP, Ducoli P, Lombardo M (2012) Optimal parameters to improve the interface quality of the flap bed in femtosecond laser-assisted laser in situ 
keratomileusis. J Cataract Refract Surg 38:1453-1459

10. De Santo M, Lombardo M, Serrao S (2004) Atomic Force Microscopy in Ophthlamic Surgery. 4th IEEE Conference on Nanothechnology

11. Serrao S, Lombardo M, De Santo MP, Lombardo G, Schiano Lomoriello D, Ducoli P, Stirpe M (2012) Femtosecond laser photodisruptive effects on the posterior human corneal stroma investigated with atomic force microscopy.Eur J Ophthalmol. ; 22 Suppl 7:S89-97

12. Nogradi A, Hopp B, Revesz K, Szabo G, Bor Z, Kolozsvari L (2000) Atomic force microscopic study of the human cornea following excimer laser keratectomy. Exp Eye Res 70:363-368

13. Lydataki S, Lesniewska E, Tsilimbaris MK, Panagopoulou S, Le Grimellec C, Pallikaris IG (2002) Excimer laser ablated cornea observed by atomic force microscopy. Single Molecules 2-3:141-147

14. Lombardo M, Serrao S (2004 Sep-Oct) Smoothing of the ablated porcine anterior corneal surface using the Technolas Keracor 217C and Nidek EC-5000 excimer lasers. J Refract Surg ; ; 20(5):450-3

15. Lombardo M (2012 Dec) Surface quality of femtosecond dissected posterior human corneal stroma investigated with atomic force microscopy. Cornea 31(12):1369-1375De Santo MPLombardo G, Schiano Lomoriello D, Desiderio G, Ducoli P, Barberi R, Serrao S

16. Ganesh S, Brar S, Lenticuloschisis (2017) : A “No Dissection” Technique for Lenticule Extraction in Small Incision Lenticule Extraction. J Refract Surg. Aug 1;33(8):563-566

17. Kamiya K, Igarashi A, Hayashi K, Negishi K, Sato M, Bissen-Miyajima H (2017) ; Survey Working Group of the Japanese Society of Cataract and Refractive Surgery. A Multicenter Prospective Cohort Study on Refractive Surgery in 15011 Eyes.Am J Ophthalmol; 175:159-168

\section{Figures}

\section{Figure 1}

Mean \pm standard deviation of the root mean square (RMS) values for the FS-LASIK and the FS-LASIK and excimer group.

\section{Figure 2}

Image of 3D atomic force microscopy (AFM) topography of the stromal surface after FS-LASIK flap creation without excimer ablation.

\section{Figure 3}


Image of the AFM vertical deflection of the stromal surface after FS-LASIK flap creation without excimer ablation.

\section{Figure 4}

Image of 3D atomic force microscopy (AFM) topography of the corneal stromal surface after FS-LASIK flap creation followed by excimer laser (3.0 D myopic ablation).

\section{Figure 5}

Image of the AFM vertical deflection of the corneal stromal surface after FS-LASIK flap creation followed by excimer laser (3.0 D myopic ablation). 